actomyosin cytoskeleton and its regulation by the small GTPase Rho. An early indication that contractile force is required for fibronectin matrix assembly was the observation that, in the absence of an intact actin cytoskeleton, cells could bind fibronectin, but could not assemble the fibrils of a fibronectin matrix ${ }^{10}$. Supporting the idea that tension is critical for the assembly of fibronectin into a fibrillar matrix, it was noted that cells growing in collagen gels can assemble a fibronectin matrix only if the gels are anchored such that the cells develop isometric tension ${ }^{11}$. Two studies have shown that fibronectin fibril assembly is stimulated by Rho-mediated actomyosin contractility $^{12,13}$. In one of these studies, use of a monoclonal antibody against a conformation-sensitive site in fibronectin revealed increased binding of this antibody to fibronectin in association with cells exerting tension, and stretching of fibronectin in a cell-free system enhanced fibronectinfibronectin binding ${ }^{13}$.

The force needed to elongate FN III modules has been worked out in two different ways. Two groups used atomic force microscopy (AFM) to stretch FN III modules found in titin and tenascin ${ }^{6,7}$, and found that the force necessary to unfold the FN III $\beta$-sandwich (Fig. 1) is about 150 picoNewtons. Now Krammer et al. ${ }^{1}$ have used steered molecular dynamics (SMD) to simulate the elongation of the $\mathrm{FN} \mathrm{III}_{10}$ module. They find that the maximum force ('force peak') required to unfold the $\mathrm{FN}$ III $_{10}$ module is 1.8 nanoNewtons, but, when the differences in several parameters between the SMD simulations and AFM experiments are taken into account, the force estimates are comparable (refs 1, 6, 7; H. Erickson, personal communication).

But Krammer et al. ${ }^{1}$ have done more than simply provide a new estimate for a force peak. The sensitivity of SMD allowed them to follow the simulated elongation of the FN III module on an angstrom scale. Using the structure of $\mathrm{FN} \mathrm{III}_{10}$ as a guide, Krammer et al. were able to discover which of the seven $\beta$-strands of FN III 10 would dis- sociate from the module with each increment of elongation. They judged that the elongation process begins with the dissociation of $\beta$-strand $G$, as its hydrogen bonds with the rest of the module are broken. Then, the loop connecting $\beta$-strands $F$ and $\mathrm{G}$ is stretched. This loop contains the arginine-glycine-aspartate (RGD) motif, a 'stick' representation of which is shown in Fig. 1. This motif binds integrins - transmembrane proteins that link the cytoskeleton with the ECM - but only when it is presented in a highly bent conformation. The force peak of the unfolding coincides with the straightening of the F-G loop, indicating that cells may straighten this loop by conveying sufficient force to fibronectin. Krammer et al. call this characteristic of FN $\mathrm{III}_{10}$ a "molecular switch", because the unfolding response is predicted to occur only under specific conditions (sufficient force) and to result in a change in the behaviour of FN III $_{10}$ (reduction of binding affinity for the integrin).

Is the Krammer et al. ${ }^{1}$ estimate of the

\title{
Of jellyfish and camgaroos
}

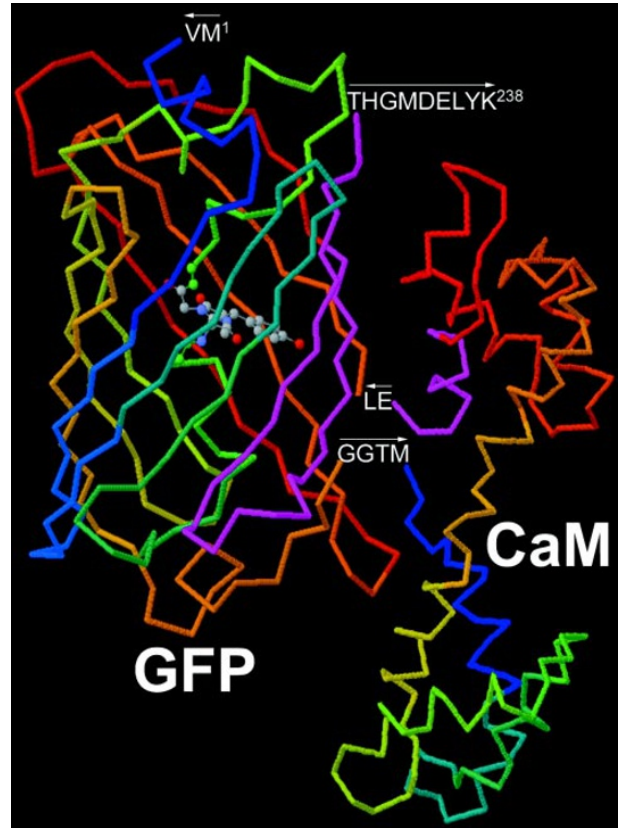

The discovery of the green fluorescent protein (GFP) from the jellyfish Aequorea victoria has revolutionized cell biological research. The ability of this protein to form a fluorescent chromophore in the absence of any additional factors has spurred wide applications of GFP as a genetically encoded marker and biosensor. GFP is used to follow cells or patterns of gene expression in living organisms or cells, and the fusion of GFP to other proteins allows researchers to monitor the whereabouts of its host proteins in virtually all subcellular compartments. Clever modifications of GFP and the use of fluorescence energy transfer (FRET) have allowed the development of blue, cyan and yellow derivatives, as well as probes that respond to changes in, for example, intracellular $\mathrm{pH}$ or calcium levels.

In the past, GFP has generally been fused in tandem to either end of its host proteins, and only in rare cases has it been inserted within its hosts. The seemingly monolithic structure of GFP, an 11-stranded $\beta$-barrel bearing the chromophore in its centre, indicated that gross alterations or insertions in GFP itself might disrupt its function. However, in a recent paper (Proc. Natl Acad. Sci. USA 96, 11241-11246; 1999), Roger Tsien and colleagues showed that such structural changes are possible, and can actually be taken advantage of, opening up new avenues for the use of GFP as a research tool.

The authors discovered that circular permutations in which the carboxy- and amino-terminal domains of GFP are swapped were tolerated, with interesting differences in their fluorescent properties. Interestingly, the authors were also able to insert an entire protein, the calcium-sensitive calmodulin (CaM), into GFP or YFP, the yellow cousin of GFP. The putative structure of a GFP-CaM hybrid is shown here. The fluorescence of this 'camgaroo' (with YFP/GFP carrying CaM in its 'pouch') increases more upon calcium binding than do any of the existing genetically encoded fluorescent probes, and is able to detect changes in calcium levels in living cells. One can now envisage inserting a wide range of sequences into GFP, such as specific cleavage or phosphorylation sites to monitor protease or kinase activities, or domains that bind ligands such as lipids, metal ions or amino acids. The future is bright, with even more exciting applications of GFP as a genetically encoded biosensor on the horizon.

BARBARA MARTE 